\title{
Numbers of Edges in Supermagic GRAPHS
}

\author{
Svetlana Drajnová, Jaroslav Ivančo, Andrea Semaničová* \\ Institute of Mathematics, P.J.Šafárik University, Jesenná 5, 04154 Košice, \\ Slovakia
}

\begin{abstract}
A graph is called supermagic if it admits a labelling of the edges by pairwise different consecutive integers such that the sum of the labels of the edges incident with a vertex is independent of the particular vertex. In the paper we establish some bounds for the number of edges in supermagic graphs.
\end{abstract}

Keywords: magic graph, supermagic graph, size of graph

\section{Introduction}

We consider finite undirected graphs without loops, multiple edges and isolated vertices. If $G$ is a graph, then $V(G)$ and $E(G)$ stand for the vertex set and edge set of $G$, respectively.

Let a graph $G$ and a mapping $f$ from $E(G)$ into positive integers be given. The index-mapping of $f$ is the mapping $f^{\star}$ from $V(G)$ into positive integers defined by

$$
f^{\star}(v)=\sum_{e \in E(G)} \eta(v, e) f(e) \quad \text { for every } v \in V(G)
$$

where $\eta(v, e)$ is equal to 1 when $e$ is an edge incident with a vertex $v$, and 0 otherwise. An injective mapping $f$ from $E(G)$ into positive integers is called a magic labelling of $G$ for an index $\lambda$ if its index-mapping $f^{\star}$ satisfies

$$
f^{\star}(v)=\lambda \quad \text { for all } v \in V(G) .
$$

\footnotetext{
*E-mail address: ivanco@science.upjs.sk, semanic@science.upjs.sk
}

This is a preprint of an article published in J Graph Theory 52: 15-26, 2006 (C) Copyright 2005 Wiley Periodicals, Inc., A Wiley Company, http://www.interscience.Wiley.com/. 
A magic labelling $f$ of $G$ is called a supermagic labelling of $G$ if the set $\{f(e): e \in E(G)\}$ consists of consecutive positive integers. We say that a graph $G$ is supermagic (magic) if and only if there exists a supermagic (magic) labelling of $G$. Note that any supermagic regular graph $G$ admits a supermagic labelling into the set $\{1, \ldots,|E(G)|\}$.

The concept of magic graphs was introduced by Sedláček [11]. The regular magic graphs are characterized in [2]. Two different characterizations of all magic graphs are given in [9] and [8].

Supermagic graphs were introduced by M. B. Stewart [12]. It is easy to see that the classical concept of a magic square of $n^{2}$ boxes corresponds to the fact that the complete bipartite graph $K_{n, n}$ is supermagic for every positive integer $n \neq 2$ (see also [12]). Stewart [13] characterized supermagic complete graphs. In [6] supermagic regular complete multipartite graphs and supermagic cubes are characterized. In [7] there are given characterizations of magic line graphs of general graphs and supermagic line graphs of regular bipartite graphs. In [10] and [1] supermagic labellings of the Möbius ladders and two special classes of 4-regular graphs are constructed. Some constructions of supermagic labellings of various classes of regular graphs are described in [5] and [6]. More comprehensive information on magic and supermagic graphs can be found in [3].

In this paper we deal with the number of edges in supermagic graphs.

\section{Number of edges}

For the number of edges in magic graphs it holds

Proposition 1. [14] A connected magic graph with $n$ vertices and $\varepsilon$ edges exists if and only if $n=2$ and $\varepsilon=1$ or $n \geq 5$ and $\frac{5 n}{4}<\varepsilon \leq \frac{n(n-1)}{2}$.

It is easy to see that all components of a magic graph are magic graphs and at most one of them is isomorphic to the complete graph $K_{2}$. Thus we have immediately

Corollary 1. A magic graph of order $n$ and size $\varepsilon$ exists if and only if $n=2$ and $\varepsilon=1$ or $n \in\{5,6\}$ and $\frac{5 n}{4}<\varepsilon \leq \frac{n(n-1)}{2}$ or $n \geq 7$ and $\frac{5 n-6}{4}<\varepsilon \leq \frac{n(n-1)}{2}$. Moreover, any magic graph with at most $\frac{5}{4} n$ edges contains a component isomorphic to $\mathrm{K}_{2}$.

The previous assertions imply the following interpolation theorem: Let $G_{1}$ and $G_{2}$ be magic graphs of order $n$. Then there exists a magic graph of order $n$ and size $\varepsilon$ for each integer $\varepsilon$ satisfying $\left|E\left(G_{1}\right)\right| \leq \varepsilon \leq\left|E\left(G_{2}\right)\right|$. A similar result for supermagic graphs is not valid. 
Theorem 1. Let $d$ be the greatest common divisor of integers $n$ and $\varepsilon$, and let $n_{1}=\frac{n}{d}$. If $n_{1}$ and $\varepsilon$ are both even, then there exists no supermagic graph of order $n$ and size $\varepsilon$.

Proof. Let $d$ denote the greatest common divisor of $n$ and $\varepsilon$ and let $n_{1}=\frac{n}{d}$, $\varepsilon_{1}=\frac{\varepsilon}{d}$. Suppose that $G$ is a supermagic graph of order $n$ and size $\varepsilon$. Then it admits a supermagic labelling $f: E(G) \longrightarrow\{a, \ldots, a+\varepsilon-1\}$ for an index $\lambda$. It holds

$$
\begin{aligned}
n \lambda & =\sum_{v \in V(G)} \sum_{e \in E(G)} \eta(v, e) f(e)=2 \sum_{e \in E(G)} f(e) \\
& =2[a+\ldots+(a+\varepsilon-1)]=(2 a+\varepsilon-1) \varepsilon .
\end{aligned}
$$

As $\varepsilon$ and $n_{1}$ are both even, the index $\lambda=\frac{(2 a+\varepsilon-1) \varepsilon}{n}=\frac{(2 a+\varepsilon-1) \varepsilon_{1}}{n_{1}}$ is not an integer, a contradiction.

For example there exists no supermagic graph of order 8 and size $\varepsilon \equiv$ 2, 4,6 $(\bmod 8)$ (i.e., with $10,12,14,18,20,22,26,28$ edges). Thus the problem to characterize the numbers of edges in supermagic graphs seems to be difficult.

Let $M(n)(m(n))$ denote the maximal (minimal) number of edges in a supermagic graph of order $n$. Evidently, $M(n)$ and $m(n)$ are not defined for $n=1,3,4$ and $M(2)=m(2)=1$. In the next sections we determine $M(n)$ and establish some bounds of $m(n)$ for $n \geq 5$.

\section{$3 \quad$ Upper bound}

Finding the maximum number $M(n)$ of edges in a supermagic graph is closely associated with the characterization of supermagic complete graphs.

Proposition 2. [13] A complete graph of order $n$ is supermagic if and only if $n=2$ or $5<n \not \equiv 0(\bmod 4)$.

Next we prove that by deleting an edge from a complete graph $K_{n}, n \geq 6$, we obtain a supermagic graph.

Theorem 2. For every positive integer $n \geq 6$, the complete graph $K_{n}$ without an edge is supermagic.

Proof. We will consider the following cases

A. $6 \leq n \not \equiv 0(\bmod 4)$. By Proposition 2 the complete graph $K_{n}$ is supermagic. Thus there exists a supermagic labelling $f: E\left(K_{n}\right) \longrightarrow$ 
$\left\{1, \ldots, \frac{n(n-1)}{2}\right\}$ for an index $\lambda$. Let $\hat{e}$ be an edge of $K_{n}$ such that $f(\hat{e})=1$. Define a labelling $g: E\left(K_{n}-\hat{e}\right) \longrightarrow\left\{1, \ldots, \frac{n(n-1)}{2}-1\right\}$ by

$$
g(e)=f(e)-1 \quad \text { for all } e \in E\left(K_{n}-\hat{e}\right) .
$$

Since $K_{n}$ is an $(n-1)$-regular graph we have

$$
g^{\star}(v)=f^{\star}(v)-(n-1) \quad \text { for all } v \in V\left(K_{n}\right) .
$$

Therefore, $g$ is a supermagic labelling of $K_{n}-\hat{e}$.

B. $8 \leq n \equiv 0(\bmod 4)$. Let $\hat{e}$ be an arbitrary edge of the complete graph $K_{n}$. Denote the vertices of $K_{n}$ by $v_{1}, \ldots, v_{n}$ in such a way that $\hat{e}=v_{n-1} v_{n}$.

Let $G$ be a subgraph of $K_{n}$ induced by the set $\left\{v_{1}, \ldots, v_{n-2}\right\}$. The graph $G$ is isomorphic to $K_{n-2}$ and by Proposition 2 there exists a supermagic labelling $f$ from $E(G)$ into $\left\{1, \ldots,\left(\begin{array}{c}n-2 \\ 2\end{array}\right)\right\}$. Clearly, $f^{\star}\left(v_{i}\right)=\left(\left(\begin{array}{c}n-2 \\ 2\end{array}\right)+1\right) \frac{n-3}{2}$ for all $i, 1 \leq i \leq n-2$.

Put a positive integer $a:=\frac{1}{4}\left[n^{3}-6 n^{2}+7 n+4\right]$ and define a mapping $g: E\left(K_{n}-\hat{e}\right) \longrightarrow\left\{a, \ldots, a+\frac{n(n-1)}{2}-2\right\}$ by

$$
g\left(v_{i} v_{j}\right)= \begin{cases}a-1+f\left(v_{i} v_{j}\right) & \text { for } 1 \leq j \leq n-2 \text { and } 1 \leq i \leq n-2, \\
a+\left(\begin{array}{c}
n-2 \\
2
\end{array}\right)-1+i & \text { for } j=n-1 \text { and } n-8 \geq i \equiv 0,3 \quad(\bmod 4), \\
& \text { or } j=n-1 \text { and } i=n-7, n-5, \\
& \text { or } j=n \text { and } n-8 \geq i \equiv 1,2 \quad(\bmod 4), \\
& \text { or } j=n \text { and } i=n-6, n-4, n-3, n-2, \\
a+\frac{(n-2)(n+1)}{2}-i & \text { otherwise. }\end{cases}
$$

A part of definition of $g$ was inspired by [4]. It is easy to see that the mapping $g$ is a bijection and for its index-mapping we get

$$
g^{\star}\left(v_{i}\right)=\frac{1}{4}\left(n^{4}-6 n^{3}+9 n^{2}+4 n-12\right) \quad \text { for } 1 \leq i \leq n .
$$

Thus, $g$ is a supermagic labelling and $K_{n}-\hat{e}$ is a supermagic graph.

It is not difficult to check that $M(5)=8$. Therefore, using previous results we get the following theorem

Theorem 3. Let $n \geq 5$ be a positive integer. Then

$$
M(n)=\left\{\begin{array}{lll}
8 & \text { for } n=5, \\
\frac{n(n-1)}{2} & \text { for } 6 \leq n \neq \equiv 0 & (\bmod 4) \\
\frac{n(n-1)}{2}-1 & \text { for } 8 \leq n \equiv 0 & (\bmod 4)
\end{array}\right.
$$




\section{Lower bound}

In this section we establish some bounds for $m(n)$. The main result is

Theorem 4. Let $n \geq 5$ be a positive integer. Then

$$
m(n) \geq 3 n-\frac{1}{2}-\sqrt{3 n^{2}-2 n+\frac{1}{4}} .
$$

Proof. Suppose that $G$ is a supermagic graph of order $n$ with $\varepsilon=m(n)$ edges. It admits a supermagic labelling $f: E(G) \longrightarrow\{a, \ldots, a+\varepsilon-1\}$ for an index

$$
\lambda=\frac{(2 a+\varepsilon-1) \varepsilon}{n} .
$$

Let $V_{3}$ denote the set of vertices of degree at least 3 , the cardinality of this set is denoted by $n_{3}$. By $n_{2}$ denote the number of 2 -vertices (i.e., vertices of degree 2). As every vertex of a supermagic graph $G$ has degree at least 2, $n=n_{2}+n_{3}$. For the number of edges we have

$$
2 \varepsilon=\sum_{v \in V(G)} \operatorname{deg}(v)=2 n_{2}+\sum_{v \in V_{3}} \operatorname{deg}(v) \geq 2 n_{2}+3 n_{3}=3 n-n_{2},
$$

thus

$$
\varepsilon \geq \frac{3 n}{2}-\frac{n_{2}}{2}
$$

If $G$ contains no 2-vertex, then $\varepsilon \geq \frac{3 n}{2}$ and the assertion is satisfied. So we can assume that $n_{2} \geq 1$.

In any supermagic graph there exists no edge joining the vertices of degree 2 , i.e. every vertex of degree 2 is adjacent to two distinct vertices of degree at least 3. It means all edges incident with the $n_{2}$ vertices of degree 2 are mutually distinct and their number is $2 n_{2}$. The sum of the labels of edges incident with 2 -vertices has to be less or equal to the sum of maximal values which can be assigned to any $2 n_{2}$ edges in the supermagic labelling $f$

$$
n_{2} \lambda \leq(a+\varepsilon-1)+\ldots+\left(a+\varepsilon-2 n_{2}\right)=\left(2 a+2 \varepsilon-2 n_{2}-1\right) n_{2} .
$$

As $n_{2} \neq 0$, by $(1)$ we get

$$
\frac{(2 a+\varepsilon-1) \varepsilon}{n}=\lambda \leq 2 a+2 \varepsilon-2 n_{2}-1 .
$$

From this inequality we yield

$$
2 n_{2} \leq 2 a\left(1-\frac{\varepsilon}{n}\right)-\frac{\varepsilon^{2}}{n}+\frac{\varepsilon}{n}+2 \varepsilon-1
$$


Any supermagic graph of order $n>2$ has more edges than vertices and so $1-\frac{\varepsilon}{n}<0$. Since $a \geq 1$,

$$
2 a\left(1-\frac{\varepsilon}{n}\right) \leq 2\left(1-\frac{\varepsilon}{n}\right)
$$

Appointing this in (3) we have

$$
2 n_{2} \leq 1-\frac{\varepsilon^{2}}{n}-\frac{\varepsilon}{n}+2 \varepsilon
$$

From (2) we get $n_{2} \geq 3 n-2 \varepsilon$, and combining it we get

$$
0 \geq \varepsilon^{2}+\varepsilon(1-6 n)+6 n^{2}-n .
$$

This inequality immediately implies

$$
\varepsilon \geq 3 n-\frac{1}{2}-\sqrt{3 n^{2}-2 n+\frac{1}{4}}
$$

which is the desired lower bound for $m(n)$.

The previous theorem implies $m(n) \geq\lceil(3-\sqrt{3}) n\rceil$. It seems that it is not possible to reach this bound. The best bound we know is $\frac{9 n}{7}$ for $n=14$, 42,70 . (We suppose that there exists an infinite family of supermagic graphs of size $\frac{9 n}{7}$.) The corresponding supermagic graph for $n=14$ is illustrated on Figure 1.

Figure 1.

Figure 2.

Now we deal with an upper bound for $m(n)$.

Let $d$ and $p$ be non-negative integers such that $k:=d+p \geq 2$. By $M_{d, p}$ denote the graph with the vertex set $\left\{u_{1}, \ldots, u_{2 k}, v_{1}, \ldots, v_{p}\right\}$ and the edge set consisting of edges

$$
\begin{aligned}
& e_{1}=u_{1} u_{2}, e_{2}=u_{2} u_{3}, \ldots, e_{2 k-1}=u_{2 k-1} u_{2 k}, e_{2 k}=u_{2 k} u_{1}, \\
& f_{1}=u_{1} u_{k+1}, f_{2}=u_{2} u_{k+2}, \ldots, f_{d}=u_{d} u_{k+d}, \\
& l_{1}=v_{1} u_{d+1}, l_{2}=v_{2} u_{d+2}, \ldots, l_{p}=v_{p} u_{d+p}, \\
& r_{1}=v_{1} u_{2 k}, r_{2}=v_{2} u_{2 k-1}, \ldots, r_{p}=v_{p} u_{2 k-p+1} .
\end{aligned}
$$


Lemma 1. The graph $M_{d, p}$ is supermagic for every odd positive integer $d$.

Proof. For every odd positive integer $d$ there exists a positive integer $s$ such that $d=2 s-1$. Put $a=p+s$. By $g$ denote the mapping from the edge set of $M_{d, p}$ to the set $\left\{a, \ldots, a+\left|E\left(M_{d, p}\right)\right|-1\right\}$ defined by

$$
g(e)= \begin{cases}a+\frac{i-1}{2} & \text { for } e=e_{i} \text { and } i=1,3, \ldots, 2 k-1, \\ a+k+\frac{i-1-d}{2} & \text { for } e=e_{i} \text { and } i=d+1, d+3, \ldots, 2 k, \\ a+k+\frac{2 k+i-1-d}{2} & \text { for } e=e_{i} \text { and } i=2,4, \ldots, d-1, \\ a+2 k-1+i & \text { for } e=r_{i} \text { and } i=1,2, \ldots, p, \\ a+3 k-i & \text { for } e=f_{i} \text { and } i=1,2, \ldots, d, \\ a+3 k+p-i & \text { for } e=l_{i} \text { and } i=1,2, \ldots, p .\end{cases}
$$

It is easy to see that $g$ is a bijection and its index-mapping $g^{\star}$ satisfies

$$
g^{\star}(v)=8 p+12 s-6 \quad \text { for every } v \in V\left(M_{d, p}\right) .
$$

Thus $g$ is a supermagic labelling and $M_{d, p}$ is a supermagic graph.

Lemma 2. For every positive integer $k \geq 2$ there exists a supermagic graph of order $3 k$ and size $4 k$.

Proof. Consider a cycle $C_{2 k}$ with vertices $u_{1}, u_{2}, \ldots, u_{2 k}$ and edges $e_{1}=u_{1} u_{2}$, $\ldots, e_{2 k-1}=u_{2 k-1} u_{2 k}, e_{2 k}=u_{2 k} u_{1}$. Let $f$ be a mapping from $E\left(C_{2 k}\right)$ to the set of positive integers defined by

$$
f(e)= \begin{cases}k-1+\frac{i-1}{2} & \text { for } i=1,3, \ldots, k, k+4, \ldots, 2 k-1 \\ 4 k-2 & \text { for } i=2 \\ k-1+\frac{i}{2} & \text { for } i=k+1 \\ 2 k-2+\frac{i-3}{2} & \text { for } i=k+2 \\ 2 k-2+\frac{i-2}{2} & \text { for } i=4,6, \ldots, k-1, k+3, \ldots, 2 k\end{cases}
$$

for $k$ odd, and

$$
f(e)= \begin{cases}k-1+\frac{i-1}{2} & \text { for } i=1,3, k+1 \\ 4 k-2 & \text { for } i=2, \\ 2 k-2+\frac{i-2}{2} & \text { for } i=k+2,2 k \\ 2 k-\frac{i}{2} & \text { for } i=4,6, \ldots, k, k+4, \ldots, 2 k-2, \\ 3 k-3-\frac{i-3}{2} & \text { for } i=5,7 \ldots, k-1, k+3, \ldots, 2 k-1,\end{cases}
$$

for $k$ even. 
Let $S_{k}$ be a graph with vertex set $V\left(C_{2 k}\right) \cup\left\{v_{1}, \ldots, v_{k}\right\}$ and edge set $E\left(C_{2 k}\right) \cup \bigcup_{i=1}^{k}\left\{v_{i} u_{i 1}, v_{i} u_{i 2}\right\}$, where $u_{i 1}, u_{i 2}$ are vertices of $C_{2 k}$ such that

$$
f^{\star}\left(u_{i 1}\right)=3 k-3+i \quad \text { and } \quad f^{\star}\left(u_{i 2}\right)=5 k-1-i .
$$

Consider a mapping $g: E\left(S_{k}\right) \longrightarrow\{k-1, \ldots, 5 k-2\}$ defined by

$$
g(e)= \begin{cases}f(e) & \text { for } e \in E\left(C_{2 k}\right) \\ 5 k-1-i & \text { for } e=v_{i} u_{i 1}, \\ 3 k-3+i & \text { for } e=v_{i} u_{i 2} .\end{cases}
$$

It is easy to check that $g$ is a bijection. Moreover its index-mapping $g^{\star}$ satisfies

$$
g^{\star}(v)=8 k-4 \quad \text { for every } v \in V\left(S_{k}\right) .
$$

Thus $g$ is a supermagic labelling and $S_{k}$ is a desired supermagic graph.

On Figure 3 there are illustrated the graphs $M_{3,0}, M_{1,3}$ and $S_{4}$ and their supermagic labellings.

Figure 3.

From previous lemmas we immediately obtain

Theorem 5. Let $n \geq 5$ be a positive integer. Then

$$
m(n) \leq\left\{\begin{array}{lll}
\frac{4 n}{3} & \text { for } n \equiv 0 & (\bmod 3) \\
\frac{4 n}{3}+\frac{5}{3} & \text { for } n \equiv 1 & (\bmod 3) \\
\frac{4 n}{3}+\frac{1}{3} & \text { for } n \equiv 2 & (\bmod 3)
\end{array}\right.
$$

Proof. It is obvious that the graphs $S_{n / 3}($ for $n \equiv 0(\bmod 3))$, the graph on Figure 2 and the graphs $M_{5,(n-10) / 3}($ for $n \equiv 1(\bmod 3))$ and $M_{1,(n-2) / 3}$ (for $n \equiv 2(\bmod 3))$ are supermagic graphs of order $n$ with the required number of edges. 
We conclude this paper with a determination of $m(n)$ for prime number $n$, but first we prove the following auxiliary result.

Lemma 3. Let $G$ be a supermagic graph of order $n \geq 5$ and size $\varepsilon$. If the greatest common divisor of the numbers $n$ and $\varepsilon$ is 1 , then $\varepsilon>\frac{4 n}{3}$. Moreover, if $\varepsilon$ is an even integer, then $\varepsilon>\frac{4 n+2}{3}$.

Proof. Consider a supermagic labelling $f: E(G) \longrightarrow\{a, \ldots, a+\varepsilon-1\}$ for an index $\lambda=\frac{(2 a+\varepsilon-1) \varepsilon}{n}$. As $n$ and $\varepsilon$ are coprime and $\lambda$ is a positive integer, then $\gamma:=\frac{2 a+\varepsilon-1}{n}$ is also a positive integer. From this we can express

$$
\begin{gathered}
\lambda=\gamma \varepsilon \\
a=\frac{1}{2}(\gamma n-\varepsilon+1)
\end{gathered}
$$

Let $n_{2}$ denote the number of 2 -vertices in $G$. Values of the edges (mutually distinct) incident with the 2 -vertices are at most $a+\varepsilon-1, a+\varepsilon-2, \ldots$, $a+\varepsilon-2 n_{2}$. Thus

$$
\lambda \leq(a+\varepsilon-1)+\left(a+\varepsilon-2 n_{2}\right)=2 a+2 \varepsilon-2 n_{2}-1 .
$$

Appointing (4), (5) in this inequality we get

$$
n_{2} \leq \frac{1}{2}((1-\gamma) \varepsilon+\gamma n) .
$$

As in the proof of Theorem 4 we get (2), and then $n_{2} \geq 3 n-2 \varepsilon$. Combining it we have

$$
(5-\gamma) \varepsilon \geq(6-\gamma) n
$$

Since $\gamma$ is a positive integer it is sufficient to consider the following cases.

A. $\gamma \geq 5$. According to Corollary 1 it yields

$$
\varepsilon>\frac{5 n}{4}=\left(1+\frac{1}{4}\right) n \geq\left(1+\frac{1}{\gamma-1}\right) n=\frac{\gamma}{\gamma-1} n .
$$

Therefore, $\varepsilon(\gamma-1)>\gamma n>\gamma n-2$. Hence $\varepsilon-2<\gamma(\varepsilon-n)=\frac{2 a+\varepsilon-1}{n}(\varepsilon-n)$. After some manipulation we have

$$
(a+\varepsilon-1)+(a+\varepsilon-2)<\frac{(2 a+\varepsilon-1) \varepsilon}{n}=\lambda .
$$

It means $n_{2}=0$, and then $\varepsilon \geq \frac{3 n}{2}>\frac{4 n+2}{3}$.

B. $\gamma \in\{3,4\}$. By (6) we get $\varepsilon \geq \frac{6-\gamma}{5-\gamma} n \geq \frac{3 n}{2}>\frac{4 n+2}{3}$.

C. $\gamma=2$. According to (6) we have $\varepsilon \geq \frac{4}{3} n$. Moreover, $\varepsilon \neq \frac{4}{3} n$. In opposite case we get $\varepsilon=4 k$ and $n=3 k$ for some integer $k>1$. It means the greatest common divisor of $n$ and $\varepsilon$ is $k$, a contradiction. Note also that (5) implies $\varepsilon=2(n-a)+1$, therefore $\varepsilon$ is an odd integer in this case.

D. $\gamma=1$. From (5) we get $\varepsilon=n-2 a+1<n$, contrary to Corollary 1 . Thus, this case is impossible. 
If $n$ is the prime number, then using Theorem 5 and Lemma 3 we immediately obtain

Theorem 6. Let $n \geq 5$ be a prime number. Then

$$
m(n)=\left\{\begin{array}{lll}
\frac{4 n}{3}+\frac{5}{3} & \text { for } n \equiv 1 & (\bmod 3), \\
\frac{4 n}{3}+\frac{1}{3} & \text { for } n \equiv 2 & (\bmod 3) .
\end{array}\right.
$$

Acknowledgement. Support of Slovak VEGA Grant 1/0424/03 is acknowledged.

\section{References}

[1] M. Bača and I. Holländer and Ko-Wei Lih, Two classes of super-magic graphs, J. Combin. Math. Combin. Comput. 23 (1997) 113-120.

[2] M. Doob, Characterizations of regular magic graphs, J. Combin. Theory, See. B 25 (1978) 94-104.

[3] J.A. Gallian, A dynamic survey of graph lebeling, Electronic J. Combinatory \# DS6 36 (2003) 53-58.

[4] T.R. Hagedorn, Magic rectangles revisited, Discrete Math. 207 (1999) 65-72.

[5] N. Hartsfield, G. Ringel, Pearls in Graph Theory (Academic Press, Inc., San Diego, 1990).

[6] J. Ivančo, On supermagic regular graphs, Mathematica Bohemica 125 (2000) 99-114.

[7] J. Ivančo and Z. Lastivková and A. Semaničová, On magic and supermagic line graphs, Mathematica Bohemica, to appear.

[8] R.H. Jeurissen, Magic graphs, a characterization, Europ. J. Combin. 9 (1988) 363-368.

[9] S. Jezný and M. Trenkler, Characterization of magic graphs, Czechoslovak Math. J. 33 (1983) 435-438.

[10] J. Sedláček, On magic graphs, Math. Slovaca 26 (1976) 329-335.

[11] J. Sedláček, Problem 27. Theory of Graphs and Its Applications, Proc. Symp. Smolenice, Praha (1963) 163-164.

[12] B.M. Stewart, Magic graphs, Canad. J. Math. 18 (1966) 1031-1059.

[13] B.M. Stewart, Supermagic complete graphs, Canad. J. Math. 19 (1967) 427438.

[14] M. Trenkler, Numbers of vertices and edges of magic graphs, Ars Combinatoria 53 (2000) 93-96. 